\title{
A CATEGORY ANALOGUE OF THE HEWITT-SAVAGE ZERO-ONE LAW
}

\author{
M. BHASKARA RAO AND K. P. S. BHASKARA RAO
}

\begin{abstract}
A category analogue of the Hewitt-Savage zero-one law is obtained in this paper.
\end{abstract}

Oxtoby [3, (3.1), p. 162] obtained a category analogue of the Kolmogorov zero-one law for measures. The main aim of this article is to obtain a category analogue of the Hewitt-Savage zero-one law. The desired result follows as a simple consequence of the theorem we prove here which is of independent interest. For details concerning the HewittSavage zero-one law for measures see Meyer [1, T4 Corollary, p. 150].

A topological space $X$ is said to be a Baire space if no nonempty open subset of $X$ is of first category. A family $\mathscr{B}$ of nonempty open sets in a topological space is called a pseudo-base if every nonempty open set contains an element of $\mathscr{B}$. In any Baire space countable intersections of dense open sets are dense. Let $X$ be any set and $G$ a group (under composition) of transformations on $X$ to $X$. A subset $A$ of $X$ is said to be invariant if $T A=A$ for every $T$ in $G$. For any $x$ in $X$, the orbit of $x$ is defined to be the set $\{T x ; T \in G\}$. A subset $D$ of a topological space is said to have the property of Baire if we can write $D=E \Delta P$, where $E$ is any open subset of $X$ and $P$ is a set of first category in $X$. A topological space $X$ is said to be homogeneous if for any two points $x$ and $y$ in $X$ there exists a homeomorphism $T$ from $X$ to $X$ such that $T x=y$.

THEOREM. Let $X$ be a topological space and $G$ a group (under composition) of homeomorphisms on $X$. In the following $(1) \Rightarrow(2) \Leftrightarrow(3) \Rightarrow(4)$. If $X$ is a Baire space, then (4) $\Rightarrow(2)$. If, further, $X$ has a countable pseudobase, then $(4) \Rightarrow(1)$.

(1) The orbit of some point $x$ in $X$ is dense in $X$.

(2) For any two nonempty open sets $U$ and $V, T U \cap V \neq \varnothing$ for some $T$ in $G$.

(3) Any nonempty open invariant subset of $X$ is dense in $X$.

Received by the editors April 8, 1973 and, in revised form, July 30, 1973.

AMS (MOS) subject classifications (1970). Primary 54A99, 57E05; Secondary 60F20.

Key words and phrases. First category sets, sets with the property of Baire, Baire space, pseudo-base, group of transformations, invariant set, Hewitt-Savage zero-one law, tail set, homogeneous spaces.

(c) American Mathematical Society 1974 
(4) Any invariant subset $D$ of $X$ with the property of Baire is either of first category or $X-D$ is of first category in $X$.

Proof. $\quad(1) \Rightarrow(2) \Leftrightarrow(3)$ are easy to prove.

$(3) \Rightarrow(4)$. Since (2) and (3) are equivalent, we use (2). Suppose $D$ is an invariant subset of $X$ with the property of Baire. Then $D=(V-P) \cup Q$, where $V$ is open in $X$, and $P, Q$ are sets of the first category in $X$. Suppose $V \neq \varnothing$ and let $U$ be any nonempty open subset of $X$. By (2), there is a $T$ in $G$ such that $T V \cap U \neq \varnothing$. Then $(X-D) \cap(T V \cap U) \subset T P$. Since $T$ is a homeomorphism, TP is of the first category in $X$. So, we have proved that given any nonempty open set $U$ in $X$ there is a nonempty open set $U^{*}$ $(=T V \cap U)$ contained in $U$ such that $U^{*} \cap(X-D)$ is of the first category in $X$. By the Banach category theorem [4, Theorem 16.1, p. 62], $X-D$ is of the first category in $X$.

$(4) \Rightarrow(2)$. Let $X$ be a Baire space. Let $U$ and $V$ be two nonempty open subsets of $X$. $\cup_{T \in G} T U$ is an open invariant subset of $X$. By (4) and the fact that $X$ is a Baire space it follows that $X-\bigcup_{T \in G} T U$ is of the first category. Since $X$ is a Baire space $\bigcup_{T \in G} T U$ is dense in $X$. Hence $T U \cap$ $V \neq \varnothing$ for some $T$ in $G$.

$(4) \Rightarrow(1)$. Let $X$ be a Baire space with a countable pseudo-base $A_{1}, A_{2}, \cdots$. It is easy to verify that $\{x$ in $X$ :orbit of $x$ is dense in $X\}=$ $\bigcap_{n \geqq 1} \bigcup_{T \in G} T A_{n}$. Since $X$ is a Baire space, $\bigcup_{T \in G} T A_{n}$ is dense open in $X$ for every $n \geqq 1$. Consequently, $\bigcap_{n \geqq 1} \bigcup_{T \in G} T A_{n}$ is a dense subset of $X$.

For a result similar to our theorem see also p. 443 of [2].

We obtain the category analogue of the Hewitt-Savage zero-one law as a corollary to the preceding theorem.

Corollary 1. Let $X$ be any topological space. Let $J$ be any infinite set. Let $Y=\prod_{\alpha \in J} X_{\alpha}$ equipped with the product topology where each $X_{\alpha}=X$. Let $G$ be the group of all transformations $T$ from $Y$ to $Y$, where each $T$ permutes finitely many coordinates of the points of $Y$ leaving the rest the same. Then any $G$-invariant subset $D$ of $Y$ with the property of Baire is either of first category or its complement is of first category. In particular, if $D$ is a tail subset of $Y$ with the property of Baire then either $D$ or $Y-D$ is of first category.

Proof. Check that condition (2) of the Theorem is satisfied for basic open subsets of $X^{J}$. The second part of the corollary follows from the fact that any tail set is $G$-invariant. For the definition of a tail set see Oxtoby [4, p. 84].

REMARK. Oxtoby [3, (3.1), p. 162] obtained the second part of Corollary 1 for any family of topological spaces $\left\{X_{\alpha}: \alpha \in J\right\}$ where each $X_{\alpha}$ admits a countable pseudo-base. In the special case where each $X_{\alpha}=X$, 
Corollary 1 is an improvement of Oxtoby's result. It would be interesting to prove Oxtoby's result dropping the countable pseudo-base condition.

Corollary 2 (H. E. White, JR.). If, for each $\alpha$ in $J, X_{\alpha}$ is a homogeneous space and $D$ is a tail set with the property of Baire in $Y=\prod_{\alpha \in J} X_{\alpha}$, then either $D$ or $Y-D$ is of first category in $Y$.

Proof. Let $\mathscr{G}_{\alpha}$ be the group of all homeomorphisms on $X_{\alpha}, \alpha \in J$. Let $I_{\alpha}$ denote the identity transformation on $X_{\alpha}$. Let $G$ be the group of all transformations $\prod_{\alpha \in J} G_{\alpha}$ from $Y$ to $Y$ where each $G_{\alpha} \in \mathscr{G}_{\alpha}$ and $G_{\alpha}=I_{\alpha}$ for all but a finite number of $\alpha$ in $J$. It is easy to check that with respect to this group $G$, condition (2) of the Theorem is satisfied and any tail set in $Y$ is $G$-invariant.

REMARK. The referee suggested Corollary 2 as an application of the Theorem. He points out that H. E. White, Jr. [5] proved this corollary in a different fashion.

ACKNOWLEDGEMENTS. In the first draft of this paper we have proved the category analogue of the Hewitt-Savage zero-one law under the assumption that $X$ is a Baire space and $J$ is countable. Professor J. C. Oxtoby pointed out reference [2] to us; this helped us to formulate the theorem of this paper for countable groups $G$, and the category analogue of the Hewitt-Savage zero-one law is deduced for countable products without any assumption on the topological space $X$. Later we succeeded in proving the category analogue of the Hewitt-Savage zero-one law for uncountable products, too. The referee pointed out that the Theorem of this paper is valid for any group G. The proof of the implication (3) $\Rightarrow(4)$ is due to the referee. We sincerely thank Professor J. C. Oxtoby and the referee for the comments and advice.

\section{REFERENCES}

1. P. A. Meyer, Probability and potentials, Blaisdell, Waltham, Mass., 1966. MR 34 \#5119.

2. J. C. Oxtoby, Note on transitive transformations, Proc. Nat. Acad. Sci. U.S.A. 23 (1937), 443-446.

3. - Cartesian products of Baire spaces, Fund. Math. 49 (1960/61), 157-166. MR 25 \#4055; 26 \#1453.

4. - Measure and category, Springer-Verlag, New York, 1970.

5. H. E. White, Jr., Two results involving Baire spaces (preprint).

Department of Probability and Statistics, The University, Sheffield S3 7RH, UNITED KINGDOM

Indian Statistical Institute, 203 B.T. Road, Calcutta 35, India 